\title{
Modelos de Picking, Routing, Layout y Slotting en la Gestión de Almacenes - una Revisión Sistemática de la Literatura
}

\section{Picking, Routing, Layout and Slotting Models in Warehouse Management - A Systematic Review}

DOI: $\underline{\text { https://doi.org/10.17981/bilo.01.01.2019.05 }}$

Fecha de Recepción:01/11/2019. Fecha de Aceptación: 02/12/2019. Fecha de Publicación: 13/12/2019

\author{
Andrés Sánchez-Comas \\ Universidad de la Costa CUC. Barranquilla, (Colombia)
}

asanchez@cuc.edu.co

\begin{abstract}
Resumen
Cuatro Bases de Datos fueron exploradas, EBSCO, SCHOLAR, IEEE Explorer y SCOPUS, con el objetivo de identificar a lo largo de la literatura modelos de Picking, Routing, Layout y Slotting que han sido propuestos como aportes a la gestión de almacenes en publicaciones de Revistas Científicas. Se describen los modelos propuestos segmentados por cada una de estas temáticas así como un breve análisis cienciométrico.
\end{abstract}

\section{Palabras clave}

Modelo, Picking, Routing, Layout, Slotting, Almacen, Revision.

\begin{abstract}
A systematic review was conducted in four databases EBSCO, SCHOLAR, IEEE Explorer, and SCOPUS with the aim of identify along the literature models about Picking, Routing, Layout and Slotting proposed as contribution in warehouses management. The proposed models are described and segmented by each one of these themes and a brief scienciometric analysis.
\end{abstract}

\section{Key Words}

Model, Picking, Routing, Layout, Slotting, Warehouse, Systematic Review.

\section{INTRODUCCIÓN}

Las operaciones de Picking, Routing, Layout y Slotting son operaciones indefectiblemente relacionadas unas con otras para lograr una óptima gestión de almacén y aún más allá de este eslabón. Las operaciones de picking por ejemplo, según [1], [2] han tomado gran importancia en la cadena de suministro, tanto en la línea de producción (líneas de ensamblaje por ejemplo), como en actividades de distribución (preparación del producto para el consumo final). La ingeniería a través de la ciencia ha realizado muchos avances para lograr puntos óptimos, es así como en la literatura se podrán encontrar de este tema, desarrollos específicos para situaciones particulares, soluciones a casos problemas, casos de estudio, y propuestas de modelos para la gestión de almacén que pueden ser aplicados y adaptados de forma genérica a necesidades específicas. Sobre este último tipo de publicaciones se centra la presente revisión. 
Aspecto importante a tener en cuenta, la cuidadosa distinción que se tuvo para seleccionar las publicaciones que harían parte de esta revisión, en cuanto a proposiciones de sistemas o desarrollos que contemplaban la utilización de modelos, mas no explicitando la formulación de un modelo como tal, sin demeritar claro está, el valor de tal aporte a la comunidad científica y la sociedad en general. Tenemos por ejemplo que [3] Propone un sistema para la formulación de planes de órdenes de picking y secuencias de manipulación por lotes a través de la integración de modelos matemáticos y técnicas de lógica difusa, dividiendo las ordenes en lotes y las prioriza la secuencia de atención para picking, lo que permite minimizar la distancia de desplazamiento. [4] propuso un enfoque para soportar el proceso de toma de decisiones en la optimización de un sistema picker-to-part, utilizando modelos analíticos y dinámicos multiparamétricos para estimar las distancias de viaje. [5] usan un modelo de simulación para analizar los efectos de la cantidad de agentes de picking y el arreglo o disposición inicial de las SKUs (stock-keeping units), finalmente provee una tabla para soportar la toma de decisiones en cuanto a estas dos variables.

La segunda sección de este artículo describe una caracterización de los artículos que conformaron la base de datos final de la revisión, en la cual se observa hacia donde están orientados los modelos, y los enfoques que cada uno pueda obtener. La tercera abre unas descripciones acerca de los modelos propuestos de Layouy, Picking, Routing y Slotting, además en unas subsecciones se muestran modelos que abarcan distintas tematicas como Picking-Layout, Picking-Routing, PickingSlotting. Finalmente se muestran las conclusiones de la revisión.

\section{CARACTERIZACION DE LAS PUBLICACIONES}

Picking y Layout parecen ser las temáticas más trabajadas, el $70 \%$ de los autores centran sus esfuerzos en estas dos tematicas casi que en proporciones iguales, sin embargo picking puntúa entre las dos y además sirve de columna para el $25 \%$ de las publicaciones que proponen modelos orientados a dos de las temáticas: Picking-Layout, Picking-Routing y Picking-Slotting. El último 5\% de las publicaciones se enfocan en Slotting. Los autores que trabajan cada una de las temáticas pueden ser apreciados en la Tabla.1.

\begin{tabular}{|c|c|c|c|c|}
\hline Autor & 烂 & $\frac{\stackrel{0}{\Xi}}{\stackrel{0}{0}}$ & 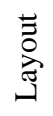 & 号 \\
\hline $\begin{array}{l}\text { (Daniels et. al. 1998) (Ashayeri et. al. 2002) (Gong et. al. } \\
\text { n.d.) (Parkish et. al. 2010) (Roodbergen et. al. 2008) } \\
\text { (Andriansyah et. al. 2010) (Xiao \& Zheng, 2011) } \\
\text { (Cardona et. al. 2015) (Roodbergen et. al. 2008) }\end{array}$ & & & $\mathrm{X}$ & \\
\hline $\begin{array}{l}\text { (Malmborg et. al. 1986) (Heung \& Cho, 2003) (Heung } \\
\text { \& Cho, 2006) (Roodbergen \& Vis, 2006) }\end{array}$ & & $X$ & $X$ & \\
\hline $\begin{array}{l}\text { (Hall, 1993) (Pandit \& Palekar, 1993) (Ventura et. al. } \\
\text { 1996) (Molnár \& Lipovszki, 2005) (Huertas et. al. 2007) } \\
\text { (Parikh \& Meller, 2009) (Parikh \& Meller, 2010) (Ji \& } \\
\text { Chen, 2010) (Feng \& Cheng, 2010) (Melacini et. al. } \\
\text { 2011) (Liu, 2012) (Hong et. al. 2012) (Li, 2012) (Zhu et. } \\
\text { al. 2012) (Rao \& Adil, 2013) }\end{array}$ & & $X$ & & \\
\hline (Jarvis \& McDowell, 1991) (Ai-Min \& Jia, 2011) & $\mathrm{X}$ & $\mathrm{X}$ & & \\
\hline $\begin{array}{l}\text { (Pan et. al. 2011) (Liu, 2011) (Jiang et. al. 2013) (Yang } \\
\text { et. al. 2015) }\end{array}$ & & $X$ & & $X$ \\
\hline (Van Oudheusden \& Zhu, 1992) (Jian \& Zheng, 2008) & & & & $X$ \\
\hline
\end{tabular}

Tabla. 1 Autores por Temática de Estudio. Fuente: Autor

Los enfoques hacia los cuales apuntan los modelos evidencia también una tendencia, el $48 \%$ de los modelos son de optimización, el $27 \%$ son modelos para la toma de decisiones, modelos para la evaluación del desempeño y análisis de costos representan $9 \%$ cada uno, mientras que el $5 \%$ son modelos para análisis de tiempos y solo uno de los modelos representa el $2 \%$ de las publicaciones enfocándose hacia modelos de predicción. La Tabla.2 permite también apreciar que los modelos parecen estar marcados principalmente hacia el mejoramiento o impacto de los desplazamientos en las bodegas, seguido de los tiempos de respuesta, costos de operación y modelos de picking. 


\section{MODELOS DE LAYOUT}

Layout, marca el inicio de los modelos, siendo la mejora de los costos de operación de la gestión del almacén el primer enfoque tratado, [6] formuló este primer modelo para la gestión de almacenes, en el cual describía los costos resultantes de distintas alternativas de layouts en almacenes que utilizaban direcciones duales para ciclos de picking, el modelo permitía evaluar además reglas comunes en almacenes donde los niveles de stocks eran determinados utilizando datos financieros. Solo diez años después se publica un segundo modelo de costos para almacenes de hospitales con el fin de minimizar los costos de operaciones anuales basado en la agrupación de suministros médicos usados por las estaciones de enfermería. De la misma forma fue hasta la próxima década que [7] propone un modelo que permitía estimar y evaluar los costos operaciones de alternativas de layouts para almacenes o centros de distribución de gran capacidad o bien con una larga variedad de productos. Siguiendo esta misma línea, cuatro décadas después es cuando a los se le incluye a los costos operaciones del layout de almacén el tema de optimización, [8] parece ser el pionero en esta incursión, presentando un método que arroja el diseño detallado en tres dimensiones $(\mathrm{x}, \mathrm{y}, \mathrm{z})$ en un almacén tipo fishbone, ademas presenta un modelo de optimización basado en algoritmos genéticos que minimiza los costos de operación total.

\begin{tabular}{|c|c|c|c|c|c|c|c|}
\hline Autor & 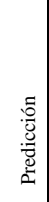 & 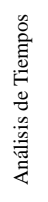 & 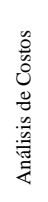 & $\mid$ & 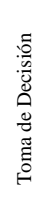 & 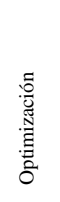 & Enfoque \\
\hline (Parkish et. al. 2010) & & & & & $\mathrm{x}$ & & Fuerza Laboral \\
\hline (Liu, 2012) & & & & & $x$ & & Metodos de Picking and Packing \\
\hline (Hall, 1993) & & & & & $x$ & & Estrategias de Picking \\
\hline (Liu, 2011) & & & & & & $x$ & Costo de Inventario \\
\hline $\begin{array}{l}\text { (Heung \& Cho, 2003) (Heung \& Cho, 2006) } \\
\text { (Pan et. al. 2011) }\end{array}$ & & & & $x$ & & & Modelos de Picking \\
\hline $\begin{array}{l}\text { (Malmborg et. al. 1986) (Ventura et. al. 1996) (Huertas } \\
\text { et. al. 2007) }\end{array}$ & & & $\mathrm{x}$ & & & & \multirow{2}{*}{ Costos de Operación } \\
\hline $\begin{array}{l}\text { (Melacini et. al. 2011) } \\
\text { (Cardona et. al. 2015) }\end{array}$ & & & & & & $\mathrm{x}$ & \\
\hline (Andriansyah et. al. 2010) & $\mathrm{x}$ & & & & & & \multirow{4}{*}{ Tiempos de Respuesta } \\
\hline (Pandit \& Palekar, 1993) (Ashayeri et. al. 2002) & & $x$ & & & & & \\
\hline (Van Oudheusden \& Zhu, 1992) & & & & & $\mathrm{x}$ & & \\
\hline $\begin{array}{l}\text { (Ji \& Chen, 2010) } \\
\text { (Ai-Min \& Jia, 2011) }\end{array}$ & & & & & & $x$ & \\
\hline $\begin{array}{l}\text { (Daniels et. al. 1998) (Melacini et. al. 2011) (Parikh \& } \\
\text { Meller, 2009) } \\
\text { (Parikh \& Meller, 2010) (Hong et. al. 2012) (Molnár \& } \\
\text { Lipovszki, 2005) (Rao \& Adil, 2013) }\end{array}$ & & & & & $\mathrm{x}$ & & \multirow[b]{2}{*}{ Desplazamiento } \\
\hline $\begin{array}{l}\text { (Jarvis \& McDowell, 1991) } \\
\text { (Roodbergen \& Vis, 2006) (Roodbergen et. al. 2008) (Jian } \\
\text { \& Zheng, 2008) (Feng \& Cheng, 2010) (Xiao \& Zheng, } \\
\text { 2011) (Li, 2012) } \\
\text { (Zhu et. al. 2012) } \\
\text { (Jiang et. al. 2013) (Yang et. al. 2015) (Gong et. al. }\end{array}$ & & & & & & $\mathrm{x}$ & \\
\hline
\end{tabular}

Tabla. Autores por Enfoques y Objetivos. Fuente: Autor

La optimización del Layout en los almacenes como tema único parece ser de trato reciente, buscando impactar el desplazamiento dentro del almacén [9] son los primeros en plantear modelo para la optimización del layout y volumen promedio de distribución basado en el principio de costo mínimo y fuerzas de interacción máximas. [10] es otro autor que en la misma línea propone un modelo matemático para minimizar las rutas para la asignación de almacenamiento de múltiples materiales en una línea de ensamblaje mixta.

El tiempo de respuesta ha buscado ser impactado también en Layout, la década de los noventa fue la espectadora del primer modelo, con un enfoque de análisis de tiempos [11], plantearon un modelo teórico de colas de un almacén rectangular con un Sistema de manejo de material multivehículo con el fin de plantear el efecto del diseño de un almacén en el tiempo de respuesta. De igual forma [12] propuso un modelo analítico que permitía calcular los ciclos de tiempo esperados para una maquina $\mathrm{S} / \mathrm{R}$ (storage/retrieval) en estructuras de estantes en zonas de almacenamiento predefinidas. 


\section{A. Layouts y Picking}

Modelos que relacionaran directa y explícitamente Layouts y Picking, fueron propuestos bajo un enfoque de optimización para el desplazamiento al interior del almacén, [13] proponen un modelo estocástico para la ubicación óptima de un producto para ordenes de picking específicos. El modelo de [14] busca minimizar la distancia de desplazamiento para ordenes de picking utilizando formulas analíticas que calculan el promedio de la distancia de una ruta de picking bajo varias políticas de ruteo. Un año después los mismos autores implementan el un modelo para minimizar las distancias de desplazamiento en un área de picking identificando una estructura de layout basado en uno o más bloques de pasillos paralelos utilizando una sola política de ruteo [15]

\section{MODELOS DE PICKING}

El 65\% de los modelos está relacionado con Picking, y en donde se encuentra el único modelo de predicción orientado a los tiempos de respuesta, [16] propuso un modelo para predecir tiempos de flujo en estaciones de picking automatizadas en distribuciones de final de pasillo, de igual forma pero buscando ahora optimizar los tiempos de respuesta, [17] proponen un modelo matemático que minimiza el tiempo de operación de un lane stacker, obteniendo el menor tiempo de desplazamiento. En la misma línea de optimización, pero con mira en los costos, [18] plantea un modelo de almacén automatizado para la toma de decisiones de picking and packing para órdenes de distinto tamaño en el sector de eCommerce, que minimiza los costos de inventarios. Para picking la optimización de costos de operación fue trabajado por [1] quien propuso un framework para minimizar los costos generales de picking manteniendo los niveles de servicios requerido como por ejemplo el tiempo de procesamiento, entendiendo por framework como un "Compendio de herramientas, estándares, buenas prácticas y fundamentos que se aplican a la solución de problemáticas específicas que pueden ser adaptados a necesidades particulares de las organizaciones" [19]

La optimización en picking también tuvo impacto en los desplazamientos, [20] formularon un modelo matemático y una heurística de algoritmos para un sistema que de asignación correlacionado para sistema de almacenamiento de productos con demandas dependientes, con el fin de minimizar las zonas de visitas en un sistema de picking en una línea de producción y debido a la rigurosidad de la metodología de revisión implementada, pese a que no data fecha, se consideró igual de importante mencionar el modelo propuesto por [21] quienes proponen y evalúan el desempeño de un modelo heuristico para el problema de picking con reposición, comparan un caso de un agente que recoge y repone versus agentes que recogen y reponen por separados.

La mejora del desplazamiento en picking también se ha desarrollado desde el enfoque de la toma de decisión, [22] fue el primero en proponer un modelo de forma simultanea se determina la asignación y secuencia de toma de decisiones en áreas de picking, 11 años después [23] desarrollaron unos modelos analíticos para estimar lotes en sistemas de operación por picking, que tiene pasillos de picking lo suficientemente anchos para permitir que el personal circule en ellos simultáneamente. [24] plantearon posteriormente un modelo empírico y matemático para determinar bloques de trabajadores en pasillos estrechos. [25] propone un procedimiento para una solución heurística para un problema de picking por lotes, adaptable a una gran cantidad de situaciones de órdenes de picking con pasillos paralelos y finalmente es [26] quien propone modelos de trayectoria para pasillos de picking multi-productos con una densidad de distribución dada. De igual forma propone en la misma publicación el segundo framework encontrado en esta revisión, el cual permite obtener tamaños de listas de picking, numero de pasillos, y tipos de frontera de almacén.

Se encuentra interesante que picking es el único de los cuatro temas tratado en esta revisión que ha contemplado modelos de evaluación de del desempeño, orientados estrategias de picking. [27] plantearon un modelo de evaluación del desempeño para sistemas de picking en Centros de Distribucion (CD) el cual es complementado con una publicación realizada tres años más tarde, que contempla para el modelo, parametros operacionales como el tamaño del almacén, tamaño de la estantería, numero de medios de transporte, y el desempeño del sistema [28]. Se consideró pertinente pese a que no plantea un modelo, la proposición de [29] ya que sus reglas de oro para la selección de estrategias de OPS (Order Picking Systems).

El siguiente año se propone un modelo de optimización simultanea que minimiza el lapso máximo de recogida en la zona de picking, a través de un Algoritmo SAC para Slotting basado en las correlaciones de los SKU y un Algoritmo NFDP para empaquetado basado en la proximidad de los SKU desarrollados por los autores.

\section{B. Picking y Routing}

Solo dos modelos que relacionan directa y explícitamente también, picking y routing fueron hallados. [30] son los primeros en plantean un modelo de planeación y asignación de órdenes de picking. Seis años después, Ai-Min \& Jia plantearon un modelo multiobjetivo para mejorar la eficiencia del movimiento de los productos dentro y hacia fuera de la bodega, almacenar productos relacionados juntos y disminuir el tiempo de transporte de los productos, al mismo tiempo de optimiza el slotting del almacén. 


\section{Picking y Slotting}

Modelos de picking y slotting también fueron hallados, todos propuestos en la década pasada [31] propone un modelo analítico de proximidad basado en probabilidad y teoría de redes de cola para la evaluación del desempeño de sistemas picker-to-part. En el mismo año [17] propone un modelo matemático no linear para determinar el volumen óptimo a alojar en cada SKU con el fin de minimizar costos de inventario. [32] por su parte, propone un método para optimizar la distribución interna de un almacén tipo fishbone combinado con estantería Drive-In, el método utiliza un modelo para el cálculo del Angulo, y un modelo para minimizar la distancia de desplazamiento. Recientemente [33] propuso un modelo de optimización de slotting para multi-shuttle AS/RS considerando una operación simultanea de almacenamiento y recuperación, con el fin de minimizar el tiempo de desplazamiento

\section{MODELOS DE SLOTTING}

Modelos de slotting fueron encontrados pocos, además de los que ya fueron mencionados anteriormente, estos buscan mejorar estrategias enfocadas solo a slotting. Desde la toma de decisiones, [34] propusieron una metodología para la distribución de los estantes basado en órdenes recurrentes que representaban un alto índice de facturación, y [35] plantearon un modelo que relaciona los productos y la frecuencia de consumo como base de una optimización multiobjetivo para la asignación de lugares de almacenamiento en almacenes en reacondicionamiento.

\section{CONCLUSIONES}

Desde los años noventa se han venido desarrollando modelos para el mejoramiento de estrategias de picking, layout, routing y slotting. La mayoría de estos modelos se orientan a optimización y toma de decisiones. La presente revisión realiza un importante aporte a la comunidad científica ya que sirve de carta de navegación para explorar estrategias de mejoramiento en estas temáticas, en busca de una óptima gestión de almacén.

\section{REFERENCIAS}

[1] Melacini, M., Perotti, S., \& Tumino, A. (2011). Development of a framework for pick-and-pass order picking system design. International Journal of Advanced Manufacturing Technology, 53(9-12), 841-854. http://doi.org/10.1007/s00170-010-2881-2

[2] Landinez-Lamadrid, D. C., Ramirez-Ríos, D. G., Neira Rodado, D., Parra Negrete, K., \& Combita Niño, J. P. (2017). Shapley Value. its algorithms and application to supply chains. INGE CUC, 13(1), 61-69. https://doi.org/10.17981/ingecuc.13.1.2017.06

[3] Lam, C. H. Y., Choy, K. L., Ho, G. T. S., \& Lee, C. K. M. (2013). An order-picking operations system for managing the batching activities in a warehouse. International Journal of Systems Science, 45(6), 1283-1295. http://doi.org/10.1080/00207721.2012.761461

[4] Manzini, R., Gamberi, M., Persona, A., \& Regattieri, A. (2007). Design of a class based storage picker to product order picking system. International Journal of Advanced Manufacturing Technology, 32(7-8), 811-821. http://doi.org/10.1007/s00170-005-0377-2

[5] Kelly, C. G., Nem-, J. R., Dalmat, D., Dean, D. P., Griffin, R. E., \& Krieger, K. L. (2014). The number of pickers and stock-keeping unit arrangement on a unidirectional picking line. South African Journal of Industrial Engineering, 25(November), 1362-1363.

[6] Malmborg, C. J., Balachandran, S., \& Kyle, D. M. (1986). A model based evaluation of a commonly used rule of thumb for warehouse layout. Applied Mathematical Modelling, 10(2), 133-138. http://doi.org/10.1016/0307$\underline{904 X(86) 90085-5}$

[7] Huertas, J. I., Díaz Ramírez, J., \& Trigos Salazar, F. (2007). Layout evaluation of large capacity warehouses. Facilities, 25(7/8), 259-270. http://doi.org/10.1108/02632770710753307

[8] Cardona, L. F., Soto, D. F., Rivera, L., \& Martínez, H. J. (2015). Detailed design of fishbone warehouse layouts 
BILO Vol. 1 no. 1, Julio - Diciembre de 2019

with vertical travel. International Journal of Production Economics. http://doi.org/10.1016/i.iipe.2015.03.006 
[9] Feng, M., \& Cheng, Y. R. (2010). Research on warehouse layout optimization and freight volume distribution of steel enterprises. Wuhan Ligong Daxue Xuebao/Journal of Wuhan University of Technology, 32(11). http://doi.org/10.3963/j.issn.1671-4431.2010.11.031

[10] Zhu, L., He, Q., \& Fan, X. (2012). Optimization of warehouse items layout in a mixed assembly workshop. Zhongguo Jixie Gongcheng/China Mechanical Engineering, 23(22), 2708-2713. http://doi.org/10.3969/i.issn.1004-132X.2012.22.013

[11] Pandit, R., \& Palekar, U. S. (1993). Response time considerations for optimal warehouse layout design. Journal of Engineering for Industry, 115(3), 322-328. Retrieved from http://www.scopus.com/inward/record.url?eid=2$\underline{\mathrm{s} 2.0-0027641958 \& \text { partnerID=tZOtx3y1 }}$

[12] Ashayeri, J., Heuts, R. M., Valkenburg, M. W. T., Veraart, H. C., \& Wilhelm, M. R. (2002). A geometrical approach to computing expected cycle times for zonebased storage layouts in AS/RS. International Journal of Production Research, 40(17), 4467-4483. http://doi.org/10.1080/00207540210153901

[13] Jarvis, J. M., \& McDowell, E. D. (1991). Optimal product layout in an order picking warehouse. IIE Transactions (Institute of Industrial Engineers), 23(1), 93-102. Retrieved from http://www.scopus.com/inward/record.url?eid=2-s2.0-0026121351\&partnerID=tZOtx3y1

[14] Roodbergen, K. J., Sharp, G. P., \& Vis, I. F. a. (2008). Designing the layout structure of manual order picking areas in warehouses. IIE Transactions (Institute of Industrial Engineers), 40(11), 1032-1045. http://doi.org/10.1080/07408170802167639

[15] Roodbergen, K. J., \& Vis, I. F. a. (2006). A model for warehouse layout. IIE Transactions, 38(10), 799-811. http://doi.org/10.1080/07408170500494566

[16] Andriansyah, R., Etman, P., \& Rooda, J. (2010). Aggregate modeling for flow time prediction of an end-of-aisle order picking workstation with overtaking. Winter Simulation Conference, 2070-2081.

http://doi.org/10.1109/WSC.2010.5678865

[17] Ji, S. Y., \& Chen, H. S. (2010). Optimization for order picking of the automated warehouse based on ant colony algorithm. Wuhan Ligong Daxue Xuebao/Journal of Wuhan University of Technology, 32(23), 69-71. http://doi.org/10.3963/j.issn.1671-4431.2010.23.018

[18] Liu, S. (2012). An Integrated Model of Picking and Packing Functions of Automated Warehouse System Based on Culture Algorithm. INTERNATIONAL JOURNAL ON Advances in Information Sciences and Service Sciences, 4(20), 229-240. http://doi.org/10.4156/aiss.vol4.issue20.28

[19] Sanchez, A., Neira, D., \& Cabello, J. (2016). Frameworks applied in Quality Management - A Systematic Review. Revista Espacios, 37(23)

[20] Xiao, J., \& Zheng, L. (2011). Correlated storage assignment to minimize zone visits for BOM picking. The International Journal of Advanced Manufacturing Technology, 61(5-8), 797-807. http://doi.org/10.1007/s00170011-3740-5

[21] Gong, J., Ota, J., Tamura, H., \& Higashi, T. (n.d.). 2A1-1 A Model and Efficient Heuristics of Order-Picking with-Replenishment in a Warehouse. Retrieved December 14, 2015, from http://nn-research.com/?a=KAV102425

[22] Daniels, R. L., Rummel, J. L., \& Schantz, R. (1998). A model for warehouse order picking. European Journal of Operational Research, 105(1), 1-17. Retrieved from http://www.scopus.com/inward/record.url?eid=2-s2.00031996750\&partnerID=tZOtx3y1

[23] Parikh, P. J., \& Meller, R. D. (2009). Estimating picker blocking in wide-aisle order picking systems. IIE Transactions (Institute of Industrial Engineers), 41(3), 232-246.http://doi.org/10.1080/07408170802108518 
[24] Parikh, P. J., \& Meller, R. D. (2010). A note on worker blocking in narrow-aisle order picking systems when pick time is non-deterministic. IIE Transactions (Institute of Industrial Engineers), 392-404.

http://doi.org/10.1080/07408170903171043

[25] Hong, S., Johnson, A. L., \& Peters, B. a. (2012). Large-scale order batching in parallel-aisle picking systems. IIE Transactions (Institute of Industrial Engineers), 44(2), 88-106. http://doi.org/10.1080/0740817X.2011.588994

[26] Rao, S. S., \& Adil, G. K. (2013). Optimal class boundaries, number of aisles, and pick list size for low-level order picking systems. IIE Transactions (Institute of Industrial Engineers), 45(12), 1309-1321. http://doi.org/10.1080/0740817X.2013.772691

[27] Hwang, H. S., \& Cho, G. S. (2003). A performance analysis of transporters for order picking warehouse design. International Journal of Industrial Engineering: Theory Applications and Practice, 10(4), 614-620. Retrieved from http://www.scopus.com/inward/record.url?eid=2-s2.0-1942453872\&partnerID=tZOtx3y1

[28] Hwang, H. S., \& Cho, G. S. (2006). A performance evaluation model for order picking warehouse design. Computers \& Industrial Engineering, 51(2), 335-342. http://doi.org/10.1016/j.cie.2005.10.002

[29] Hall, R. W. (1993). Distance approximations for routing manual pickers in a warehouse. IIE Transactions (Institute of Industrial Engineers), 25(4), 76-87. Retrieved from http://www.scopus.com/inward/record.url?eid=2s2.0-0027630124\&partnerID=tZOtx3y1

[30] Molnár, B., \& Lipovszki, G. (2005). Multi-objective routing and scheduling of order pickers in a warehouse. International Journal of Simulation: Systems, Science and Technology, 6(5), 22-32.

[31] Pan, L., Huang, J. Z., \& Chu, S. C. K. (2011). Order Batching and Picking in a Synchronized Zone Order Picking System. Proceedings of the International Conference on Industrial Engineering and Engineering Management, $156-160$.

[32] Van Oudheusden, D. L., \& Zhu, W. (1992). Storage layout of AS/RS racks based on recurrent orders. European Journal of Operational Research, 58(1), 48-56. http://doi.org/10.1016/0377-2217(92)90234-Z

[33] Xiao, J., \& Zheng, L. (2008). Slotting optimization model for overhaul warehouses. Qinghua Daxue Xuebao/Journal of Tsinghua University, 48(11), 1883-1886. Retrieved from http://www.scopus.com/inward/record.url?eid=2-s2.0-57649098350\&partnerID=tZOtx3y1

[34] Jiang, M. X., Feng, D. Z., Zhao, Y. L., \& Yu, M. F. (2013). Optimization of logistics warehouse layout based on the improved Fishbone layout. Xitong Gongcheng Lilun Yu Shijian/System Engineering Theory and Practice, 33(11), 2920-2929. Retrieved from http://www.scopus.com/inward/record.url?eid=2-s2.084891643846\&partnerID=tZOtx3y1 